\title{
Le sujet entre sidération et jouissance: I'issue sublimatoire. À propos de l'œuvre de Francisco Brennand
}

\author{
Véronique Donard*1
}

Cet article traite de l'univers inconscient des sculptures de l'artiste brésilien Francisco Brennand (1927) et des mécanismes archaïques qui sous-tendent leur création. Axée sur la thématique de l'origine, cette approche des ouvres de Brennand étudie les racines du désir sexuel et son rapport aux objets partiels, considérant la sidération du sujet face à la cruauté d'une sexualité archaïque et la jouissance de l'artiste comme défi à la mort et à l'énigme du néant. Elle s'intéresse alors au processus sublimatoire comme transmutation du matériel traumatique et au destin de la pulsion scopique et de cruauté dans la création artistique.

Mots clés: Brennand, sublimation, origine, mythe, jouissance, pulsion scopique et de cruauté

${ }^{* 1}$ Universidade Católica de Pernambuco - Unicap (Recife, PE, Br). 
Francisco de Paula de Almeida Brennand, né en 1927, est un sculpteur, peintre et céramiste brésilien. Bien que sa renommée soit acquise au Brésil, ses œuvres, jugées trop crues ou trop érotiques, sont souvent mal comprises et parfois mal reçues. De fait, admiré, décrié, attaqué ou encensé, il ne laisse personne indifférent face à sa prolifique production artistique. Travaillant actuellement à l'étude de son œuvre, je traiterai ici de l'univers inconscient qui émane de ses sculptures, dont le nombre avoisine les 2000, et des mécanismes archaïques qui, chez Brennand, commandent le geste créateur. Les propos de l'artiste qui seront ici communiqués sont tirés d'une première série d'entretiens, réalisés en 2012.

Au centre d'une propriété familiale située aux alentours de la ville de Recife, état de Pernambouc, nord-est du Brésil, Francisco Brennand a érigé un monde de rêves et de tourments, atelier-musée connu sous l'appellation de «Oficina Brennand». C'est en 1971 qu'il s'installe dans la fabrique de céramiques en ruines que son père avait fondée en 1917. Commence alors une longue reconstruction, entièrement pensée comme une seule et unique œuvre, sorte d'ode à la gloire d'un père déchu qu'il ne cessera de réériger en totem et à une mère-nature-vénus glorifiée, exaltation qui permet à l'artiste de pleurer la mère prisonnière, malheureuse et enchaînée. Cet attachement à son enfance et à ses parents ponctue son discours, labile et cordial, Brennand semblant tenir à tisser des liens de causalité qui expliqueraient en partie sa frénésie créatrice. Les larmes de sa mère et l'image d'un père pionnier dans l'industrie de la céramique, meurtri et castré par son histoire personnelle, semblent hanter encore aujourd'hui le cœur de l'artiste, comme si l'enfant qu'il était n'avait pu trouver réponse aux scènes auxquelles il assistait. 
Ces réponses, Brennand les cherche dans le processus de création mais également dans le regard que le public porte sur ses œuvres. Il se promène ainsi dans son atelier aux heures d'ouverture, observe les visiteurs, accepte de façon cordiale de signer des autographes et de poser pour des photos souvenirs, mais, surtout, écoute avec attention leurs critiques et remarques, notant intérieurement celles qui pourront l'aider à mieux se comprendre.

\section{À l'origine, la forme}

«Le futur a un cœur ancien», répète-t-il inlassablement, citant l'écrivain italien Carlo Levi. Cela explique en partie la récurrence des thèmes mythologiques dans ses productions, ainsi que la force que la thématique de l'origine, de façon générale, imprime à son univers artistique.

Le visiteur qui pénètre dans la propriété est d'emblée confronté à une œuvre foisonnante et colossale, dédiée à la terre-mère et à la création. Sous l'œil attentif d'un sorcier énigmatique, entouré d'animaux et d'une armée d'oiseaux-rocs, vautours légendaires, se dresse au centre un temple qui abrite sous sa voûte l'œuf primordial, matrice de toutes les formes. «Pauvre de la forme qui ne peut tenir dans un œuf!», s'exclame Brennand. De fait, les formes arrondies de ses sculptures, quoi qu'elles représentent, semblent une infinie et kaléidoscopique déclinaison de cette forme principielle. Il est par ailleurs significatif que la biologiste Cecilia Toro se soit passionnée par ses sculptures, sidérée d'y retrouver les formes des micro-organismes qu'elle avait l'habitude d'étudier au microscope (Toro, 2001) Brennand en étant par ailleurs le premier surpris. Il eu la sensation d'avoir avec son œuvre touché quelque chose de juste.

Cet attrait de l'artiste pour la thématique de la forme originaire n'est pas sans nous rappeler le Timée de Platon, où le philosophe s'attache à expliquer non seulement la génération et la corruption, d'un côté, et la stabilité, de l'autre, mais aussi l'unité du monde et sa multiplicité. Car, si pour Platon les choses sensibles diffèrent des formes intelligibles et sont multiples et distinctes, il est nécessaire qu'elles aient un «lieu», un espace matriciel que le disciple de Socrate nomme la chôra, où elles puissent gagner matière et forme propres, ce même lieu où elles reçoivent le sceau formel intelligible qui leur confère leur unité dans la diversité. Cependant, pour que le changement n'intervienne pas dans le monde de façon chaotique, il faut qu'il y ait une volonté extérieure qui puisse imprimer à la gestation du réel et au mouvement une mesure et un ordre précis. Platon émet donc l'hypothèse d'un démiurge qui donnerait à la création cette ordonnance harmonique, en en faisant un «cosmos» (bon ordre, arrangement) et non pas un 


\section{ARTIGOS}

«chaos» (gouffre, abîme, ténèbres). L'harmonie ainsi engendrée par le juste rapport des contraires - le Même et l'Autre - au centre de cet être parfait qu'est le monde confère à ce dernier une stabilité qui lui permet de se tenir seul par soi-même, structuration interne que Platon qualifie de «philia» (amitié).

Ce parallèle avec la pensée de Platon pourrait donner à penser que l'artiste croirait œuvrer tel un démiurge, aux prises avec la nature brute et la matière, pour donner à celles-ci un sens et un agencement ordonné. Même si cette image n'est pas tout à fait inexacte, ce serait mal connaître Brennand que de réduire ses sculptures à la tentative d'un réagencement harmonieux de l'univers, dont il serait le seul maître d'œuvre. Pour ce sculpteur et céramiste, la fonction de créateur est en dernier lieu assumée par le feu. Il guette ainsi la sortie du four de ses sculptures avec anxiété et excitation, s'apprêtant à découvrir le travail transformateur de cette fournaise auquel il ne fait que collaborer. Il expérimente ainsi des cuissons successives, parfois par dizaines, pour parfaire une technique qui accorde au feu le rôle principal, car seul ce dernier peut porter une œuvre à sa perfection.

Cela étant, l'œuf primordial, emblème de l'éternité et de l'immortalité, couve également, explique-t-il, la gestation du mal radical, ce qui nous renvoie à l'harmonie des contraires empédoclienne reprise par Platon, où encore à la coincidentia oppositorum omniprésente dans les mythes de création. Cette volonté de représenter une réalité ambivalente, duelle, est en effet constante dans son œuvre. La présence du mal, larvé, que l'on peut pressentir sans pouvoir le cerner, ce mal qui pointe progressivement sa tête est symbolisé de façon récurrente par un serpent qui perce la coquille de son œuf. Car, nous dit l'artiste, le sentiment qui préside son œuvre, c'est la peur, la même terreur, dit-il, ressentie par Gogol... La peur de l'énigme, la peur de la mort, la peur de soi...

Toutefois, les sculptures censées représenter cette gestation du mal ne sont pas effrayantes en elles-mêmes. La persistance des formes arrondies, la douceur de la céramique émaillée, le regard même, inoffensif, de ces serpents qui pointent leur tête hors de l'œuf, ôtent à ces derniers toute dangerosité apparente.

Cette peur, le visiteur ne la ressent donc pas, mais la sidération et le malaise l'envahiront progressivement, sournoisement, lorsque ses yeux découvriront autant de cloaques béantes, des serpents qui sont autant de pénis, d'excréments généreux et bien moulés... Si «les choses sont éternelles parce qu'elles se reproduisent», ${ }^{1}$ «le sexe, plus que la mort, laisse l'être humain face à une perplexité inguérissable», ${ }^{2}$ dira-t-il à un journaliste.

${ }^{1} \mathrm{http}: / /$ www.youtube.com/watch?v=ewrrUKXwq94

${ }^{2} \mathrm{http}: / /$ www.eluniversal.com.mx/notas/841958.html 


\section{La perplexité face au sexuel}

Car parler de l'œuvre de Brennand sans souligner l'omniprésence des thématiques sexuelles serait un non-sens, ou plutôt un non-voir, un évitement de la gêne que le cru, voire le cruel de la sexualité peut causer chez le public. De fait, l'univers sexualisé à l'extrême de Brennand provoque fascination, excitation, rires moqueurs ou gênés, mais également horreur et dégoût, que l'artiste reconnaît ressentir lui-même, soutenant que son œuvre n'a rien d'érotique. Il cite ainsi presque avec reconnaissance les termes qu'une vieille dame, qu'il nomme en souriant sa «première véritable critique d'art», avait proféré, horrifiée, lors de sa visite: «C'est une boucherie!» Complaisance dans l'horreur? Non, sidération. Sidération que l'artiste réussit à communiquer en exposant son questionnement principiel au public, comme si cette contagion par le voir était un moyen de s'en débarrasser, de s'en soulager momentanément, mais, peut-être aussi, d'y trouver une réponse dans le regard de l'autre. Plaisir de «voir-montrer» à l'œuvre dans la pulsion scopique qui l'anime, ses sculptures sont destinées à être vues, accueillies par le regard de l'autre sans lequel elles resteraient incomplètes. Il regrette ainsi que le visiteur ne lui fasse jamais de commentaires sur une œuvre en particulier, mais toujours sur sa production perçue comme un tout. Or, chaque sculpture enferme une énigme qui lui est propre, une histoire, un moment, et apparaît aux yeux de l'artiste comme un univers en soi.

Reprenons notre visite. Encore sous la fascination de l'extérieur rythmé et foisonnant de l'Oficina Brennand, le visiteur, au fur et à mesure de son avancée, se retrouve donc face à des sculptures dénuées de toute pudeur. Les organes féminins sont généreux, ronds, voluptueux: arborant vulves, seins et fesses, les statues féminines trônent majestueusement, offrant dans les moindres détails au regard leur anatomie généreuse. Plus en retrait, se dresse orgueilleusement un phallus, sous la forme d'un oiseau stylisé. L'Oficina Brennand est donc un univers placé sous le signe d'Eros, vulvaire, phallique, mais cependant peu de sculptures masculines affichent la nudité ou l'érection de leur pénis, contrairement aux statues féminines qui exhibent sans honte leurs organes. Le phallus est presque toujours détaché du corps, car la statue masculine adulte, chez Brennand, ne donne pas à voir sa nudité. L'homme, dans l'univers de Brennand, semble pudique, comme en témoigne cette statue d'Adam, à taille humaine, cachant son sexe de la main... Ce que n'est certes pas l'infans, qui, lui, dévore et désire, comme en témoignent plusieurs «bébés cannibales» donnant à voir un pénis en érection qui suffit à mettre en scène leur cannibalisme inné.

Si le phallus en tant qu'objet imaginaire menacé par la castration apparaît clairement dans le discours de l'artiste - questionné sur l'apparente pudeur de 


\section{ARTIGOS}

son Adam, il explique: «J'ai craint le sectionnement de l'organe par un public malveillant», en ajoutant avec malice que «cinq doigts sont autant de pénis» - il a, dans l'Oficina Brennand, toute sa place comme signifiant de la jouissance. Cette allusion à la théorie lacanienne de la jouissance est d'autant plus pertinente que le questionnement du sculpteur sur l'être et l'existence rejoint les racines mêmes de la sexualité.

Que suis-je? Je suis à la place d'où se vocifère que "l'univers est un défaut dans la pureté du Non-Être". Et ceci non pas sans raison, car à se garder, cette place fait languir l'Être lui-même. Elle s'appelle la Jouissance, et c'est elle dont le défaut rendrait vain l'univers. (Lacan, 1960/1966, p. 819)

La place de la jouissance dans le travail créateur de Brennand fait apparaître ses productions comme un défi à la mort et à l'énigme du néant, une redistribution des règles universelles qui fixent à l'existence un début et une fin, lancé par un sujet prisonnier de l'inéluctable aimantation de la chose sexuelle. Chose sexuelle perçue en effet comme «Chose» échappant à toute compréhension, de la béance de laquelle l'artiste hurle son désir et son manque. Associés à cette expérience, la peur et la sidération qui fondent son impérieuse nécessité créatrice élargissent le champ de compréhension de sa compulsion à créer. La Chose mythique restant à jamais introuvable et inexplicable, il s'agit donc pour le sculpteur d'investir l'univers du mythe pour ne pas se laisser happer par le néant.

Cet univers à la fois mythologique et sexuel des sculptures de Brennand renvoie à la théorie des mythes magico-sexuels proposée par S. de Mijolla-Mellor (2002). Car, s'il s'agit bien ici de sexualité, il faut plutôt parler de sexualité archaïque, qui met en scène des organes qui ont une vie propre et qui sont autant d'objets partiels détaché d'un ensemble qui fait sens.

S. de Mijolla-Mellor soutient que le processus du mythe magico-sexuel fait son apparition lorsque l'activité de penser du petit enfant, à savoir l'impérieuse nécessité de tisser des liens causals, est mise en échec par un non-sens qui menace son narcissisme primaire (Ibid.). Cette activité de penser, avant de chercher à élaborer des théories, va donc tout d'abord tenter de colmater la brèche, si menaçante, par laquelle le non-sens risque d'envahir et d'anéantir la toute-puissance narcissique primaire et le sentiment de soi de l'enfant.

Le mythe magico-sexuel fait par conséquent irruption dans le travail psychique de l'enfant confronté au danger de la perte de soi, qui va mettre en place un système causal se suffisant à soi-même, "le "il y a", anhistorique, sans origine ni terme, clos sur lui-même comme le narcissisme des débuts» (S. de Mijolla-Mellor, 2002, p. 93).

C'est ainsi que Brennand expliquera: «Ici, j'ai créé une mythologie absolument particulière, personnelle, qui n'est en lien avec aucun type d'érudition. 
Je cultive les mythes, non comme un approfondissement, mais comme un artiste qui développe des idées qui surgissent et qui sont saisies à chaque moment, en comblant, également, des lacunes de l'esprit».

\section{Sacrifice et création}

Mais le constat du travail de mythes magico-sexuels dans l'univers de Brennand n'est pas le seul à éclairer son œuvre. Le meurtre, compris comme la mise à mort de la matière, et la mort en elle-même le sont aussi, tout comme le sont la terreur, la souffrance, celle du non-sens et celle de l'engendrement suivi de la joie de la découverte de sa propre création, une fois terminé et parfaite par le feu. Nous avons là une parfaite illustration du processus sublimatoire à l'œuvre dans la création artistique, proche de celui que nous pouvons observer dans l'acte d'écriture, qui apparaît comme une transmutation du matériau traumatique et de la pulsion meurtrière qui s'en dégage (Donard, 2009).

Les termes «écriture» et «sculpture» ont par ailleurs la même racine étymologique. K. Rouquet-Brutin (2005), dans son texte "Archives de la cruauté chez Patricia Cornwell», nous rappelle que la racine indo-européenne sker, qui signifie «couper, gratter, inciser», entre dans l'étymologie du verbe scribere (écrire), ce qui établit un lien significatif entre le geste de l'incision et celui de l'écriture. Également rattachés à cette étymologie sont scalpellum (lancette) et scalpere (gratter, creuser), le mot «sculpter» étant issu de ce dernier.

Le travail du feu, principal allié de notre artiste, qui transforme la matière et complète l'œuvre en la signant, renvoie ainsi à un processus sublimatoire proche de l'œuvre alchimique, qui comprenait trois étapes: la première était celle qui visait à «préparer la matière», c'est-à-dire à provoquer sa mort par solution de liquéfaction, par trituration ou putréfaction; la deuxième, la sublimation proprement dite, visait à libérer l'âme de la matière et à déployer ses potentialités; et c'est en soumettant ces dernières à un dernier processus complexe appelé «l'œuvre au rouge» que les alchimistes étaient censés obtenir la pierre philosophale.

Ce que l'analogie du processus sublimatoire alchimique avec le processus psychique du même nom nous suggère, c'est que, pour qu'il y ait sublimation, à savoir purification, volatilisation, spiritualisation de la matière, il faut préalablement qu'il y ait une mise à mort de cette même matière. Sans meurtre, donc, pas de sublimation. Pour Brennand, nul doute, sans transpercer, malaxer, frapper la matière, pas de création. Dans cet œuvre colossale, il est celui qui détruit et qui souffre; le feu est celui qui transforme, qui engendre, qui crée, et trouve une issue favorable à la perplexité du créateur. L'artiste cite ainsi volontiers Baudelaire, qui, 


\section{ARTIGOS}

dans L'Art romantique, remarquait: «C'est un des privilèges prodigieux de l'Art que l'horrible, artistiquement exprimé, devienne beauté et que la douleur rythmée et cadencée remplisse l'esprit d'une joie calme».

Mais, si meurtre il y a, il ne s'agit pas d'une destruction dénuée de sens, bien au contraire: le meurtre n'opère ici que pour qu'un sens supérieur advienne, pressenti par l'artiste et parfait par le feu. Or, tuer la matière pour la sublimer, pour en extraire son âme renvoie à la notion de «sacrifice».

La locution latine sacer facere signifie «rendre sacré». Le terme «sacrifice», qui désigne à la fois ce qui est offert et l'action d'offrir, est issu de sacrificare, qui consiste donc à rendre une chose ou un être sacrés, à fabriquer du sacré. Bien que Brennand ait également dédié un temple à la thématique du sacrifice, il ne cherche pas à «fabriquer» du sacré mais bien, comme nous l'avons déjà souligné, à sonder le mystère de la vie et de la mort, du bien et du mal, de la sexualité comme principe métaphysique suprême, en utilisant ses créations comme autant de bouteilles à la mer, autant de questions sans véritables réponses, réponses fragmentées, accordées par le travail du feu, par le regard de l'autre sur son œuvre.

\section{La pulsion scopique et de cruauté, matériau privilégié du processus sublimatoire}

Cela soulève la question du matériau psychique concerné par ce processus sublimatoire. Sans conteste, il s'agit en premier lieu d'une libido explosive, démesurée, un torrent qui jaillit sans cesse, qui n'a ni frein ni possibilité de contenance autre que son évacuation. La création apparait alors comme la seule façon d'exorciser ce fleuve continu d'énergie sexuelle, qui risquerait, s'il n'était dévié, canalisé, de déborder totalement le moi, de l'inonder...

Cependant, il ne s'agit pas de n'importe quelle libido, mais de celle qui signe une sexualité obsédée par la question de l'origine, ce qui nous ramène encore une fois à la question des mythes magico-sexuels. Il s'agit, pour l'artiste, avant tout de sonder la matrice, de retrouver le lieu mythique de l'arkhè, d'observer sans relâche les modèles, le corps féminin, ses fluides, son odeur, cherchant à y décrypter l'énigme qu'il renferme. Non, l'homme n'est pas pudique, chez Brennand, il est un seul et unique phallus dressé, cherchant obstinément sa route vers les entrailles féminines. Il est ce lieu même de la jouissance. Pas de corps pour l'homme sinon un corps torturé : lorsque la libido vient le sauver de sa condition humaine, il n'est plus qu'un organe dressé de toutes ses forces, de toute sa vigueur, pointant vers le centre de la terre, le centre de la mère, le centre de la femme que tout destine à faire jouir. Le corps de la femme est ainsi pour l'artiste un instrument que l'homme se doit 
d'apprendre à faire vibrer harmonieusement, au risque de sa propre insatisfaction, car la satisfaction de l'artiste-amant est de faire exploser l'orgasme féminin, de déchainer le volcan enfoui dans chaque femme, de faire de la jouissance de la femme son œuvre d'art.

J'ai émis ailleurs l'hypothèse que le matériau psychique d'une sublimation qui passe par la mort puisse n'être autre que celui issu du trauma, trauma originaire mais également toute situation traumatique postérieure venant se greffer sur celui-ci (Donard, 2012). Le trauma, nous le savons, engendre un remaniement pulsionnel important, suscitant notamment un véritable déferlement interne de pulsions mortifères. La seconde théorie des pulsions n'a d'ailleurs pas été pensée par Freud autrement qu'à partir du constat de la prégnance d'une compulsion de répétition, suite à des situations traumatiques non élaborées (Freud, 1920/1981).

Ce remaniement pulsionnel me semble comporter deux causes principales: d'une part, la situation traumatique rend urgent le besoin de sens; d'autre part, les pulsions mortifères doivent trouver une issue pour que la psyché retrouve une certaine homéostasie et puisse continuer à fonctionner.

Ferenczi, en pointant le rôle du clivage du moi dans la survie psychique de l'individu confronté à un trauma précoce, explique qu'une partie consciente du moi se retire de celle qui est en souffrance pour observer de loin la scène traumatique et en proposer au moi oppressé une interprétation, prenant en charge la folie (ou la dépression) environnementale. Le sujet ainsi clivé devient un être dont l'avenir est de soigner, d'écrire ou encore d'expliquer. C'est là, à proprement parler, ce que Ferenczi entendait par «nourrisson savant». Il me semble cependant que nous pouvons ajouter à ces modalités de mises en sens et d'auto-réparation l'impérieuse nécessité de créer et de recréer le monde, propre à bien des artistes.

C'est donc au moment de la gestion interne du trauma, et ce parce que la psyché possède les prérequis aptes à lui permettre d'engager ce travail, que semble s'effectuer le travail sublimatoire sur des pulsions mortifères déchainées, les liant à nouveau avec Eros, maîtrisant leur revendication destructrice, les déviant de leur but meurtrier. En nous calquant sur le paradigme sacrificiel pour penser cette sublimation, la création artistique ou littéraire serait alors une sorte d'autel sacrificiel sur lequel une victime symbolique serait offerte en lieu et place de l'être préalablement désigné - le moi ou l'objet, selon la gestion sadique ou masochique des pulsions de mort - , ce qui permet à la compulsion traumatique de s'élaborer, dans et par la combustion de l'opération sublimatoire.

La pulsion ici visée, plus que la pulsion sexuelle, semble être cet étrange amalgame pulsionnel que Freud a nommé pulsion scopique et de cruauté (Freud, 1905/1997b). Dans le cas de notre sculpteur, nous retrouvons à l'œuvre, sans nul doute, la Schau und Grausamkeit-strieb. Chaque sculpture est une façon de sonder le mystère, de percer le corps féminin, d'exposer sa nudité pour tenter de 


\section{ARTIGOS}

le comprendre. D'autres sculptures, non sexualisées, plus sombres, semblent quant à elles scruter le visage de la mort. L'omniprésence du phallus dans l'univers de l'Oficina Brennand est par ailleurs significative de cette béance à partir de laquelle l'artiste crée.

Cette pulsion a pour particularité d'être préobjectale ou anobjectale, selon D. Cupa (2002, 2005), voire désobjectalisante, pour d'autres auteurs. En effet, si la Schau und Grausamkeit-strieb est une pulsion partielle, donc sexuelle a priori, elle possède cependant, comme son nom l'indique, une composante particulièrement morbide. Il semble, en effet, que cette pulsion, qui fonde toute capacité de recherche, d'investigation, d'écriture et de lecture, ne peut être rangée uniquement du côté des pulsions sexuelles, mais condense les deux formes pulsionnelles originaires qu'elle fait travailler conjointement.

Cette hypothèse nous aiderait à cerner le lien, indubitable mais encore obscur, entre les pulsions sexuelles et les pulsions de mort au travail dans la création artistique. Est-il vraiment possible de postuler une dichotomie métapsychologique entre celles-ci, même si, dans leur application au champ phénoménal, Freud ait pris soin de préciser que l'on ne peut trouver qu'une conjugaison, voire un amalgame des deux pulsions, l'une ne procédant jamais sans l'autre? En d'autres termes: la dichotomie vie/mort est elle pertinente pour comprendre le travail artistique ou faut-il envisager une activité pulsionnelle qui mette au travail une pulsion qui soit à la fois sexuelle et mortifère? La pulsion scopique et de cruauté, comprise comme pulsion hybride, en serait alors le matériau privilégié.

Pour essayer de penser cette question, il est intéressant de noter qu'il est possible d'établir un lien entre le mouvement dérivatoire opéré par la sublimation et la perversion. En effet, comme l'explique Sophie de Mijolla-Mellor, le processus sublimatoire, lorsqu'il permet au sujet d'énoncer des certitudes qui l'assujettissent à la loi seulement en tant qu'il en est lui-même l'énonciateur, vient à côtoyer et frôler dangereusement le mécanisme pervers.

On peut de fait considérer que l'énergie libidinale, dans la perversion et dans la sublimation, opèrent toutes deux un mouvement de contournement de l'interdit et parviennent, moyennant certaines limites, non seulement à maintenir l'écoulement du flux mais à le renforcer du fait de cet obstacle. (...) Il y a deux mouvements qui sans être identiques se rapprochent: le «per-vertir» et le «de'-river» indiquent tous deux que le flux libidinal est parvenu à ne pas se laisser prendre dans la nasse du refoulement. (S. de Mijolla-Mellor, 2005, p. 28)

Nous pourrions, en ce cas, considérer la sublimation de la pulsion de la cruauté comme l'équivalent de sa perversion, œuvrant, à l'encontre de son but premier qui serait la négation de l'objet, son résultat contraire, à savoir non seulement la survie de l'objet mais son accession à une réalité et une identité nouvelles. 


\section{Conclusion}

Si la dynamique psychique propre à la création artistique est loin d'avoir livré ses secrets, la recherche tenant compte de ses déterminants archaïques s'ouvre au théoricien comme un abîme vertigineux. Toucher au processus créateur en ce qu'il a de plus enfoui et de plus mystérieux emporte le chercheur sur un continent inconnu et amoral où tout devient possible. Mythes, organes partiels, intrications pulsionnelles, perversions et sublimation, désir et jouissance se mêlent à l'histoire singulière du créateur, unique entre toutes et pourtant marquée du sceau d'une l'humanité commune. C'est sans doute pour mieux se comprendre que l'on cherche à comprendre l'autre. En ce sens, Francisco Brennand apparaît comme un interlocuteur hors pair, aussi intéressé par ce que l'autre est en soi que par ce qu'il lui apporte. Discuter longuement et profondément avec lui, sillonnant les routes de sa vie ou les méandres de sa pensée créatrice est une des grandes chances qu'il m'ait été donné, sur le plan de la recherche comme sur un plan personnel. Sa disponibilité et le sérieux avec lequel il considère son interlocuteur rendent ce dernier plus apte à le comprendre. Qu'il en soit ici remercié.

\section{References}

Cupa, D. (2002/4). La pulsion de cruauté, Revue française de psychanalyse, PUF, 66, 1073-1089.

Cupa, D. (2005). La cruauté du surmoi féminin. In S. de Mijolla-Mellor (dir.), La cruauté au féminin (pp. 55-92). Paris: PUF.

Donard, V. (2009). Du meurtre au sacrifice. Psychanalyse et dynamique spirituelle. Paris: Cerf.

Donard, V. (2012). La sublimation propre au sentiment religieux. In S. de Mijolla-Mellor (dir.), Traité sur la sublimation. Paris: PUF.

Freud, S. (1997a). Le problème économique du masochisme. In Névrose, psychose et perversion (pp. 287-297). Paris: PUF. (Trabalho original publicado em 1920)

Freud, S. (1997b). Trois essais sur la théorie sexuelle. Paris: Gallimard. (Trabalho original publicado em 1905).

Freud, S. (1981). Au-delà du principe de plaisir. In Essais de psychanalyse (pp. 47-128). Paris: Payot. (Trabalho original publicado em 1920).

Ferenczi, S. (1982a). Confusion de langue entre les adultes et l'enfant. In Euvres complètes, IV (1927-1933) (pp. 125-135). Paris: Payot. (Trabalho original publicado em 1932).

Ferenczi, S. (1982b). Réflexions sur le traumatisme. In Euvres complètes, IV (1927-1933) (pp. 139-147). Paris: Payot. (Trabalho original publicado em 1934).

Ferenczi, S. (1993). Le rêve du nourrisson savant. In Euvres complètes, III (1919-1926) (p. 203) Paris, Payot. (Trabalho original publicado em 1923). 


\section{ARTIGOS}

Lacan, J. (1966). Subversion du sujet et dialectique du désir dans l'inconscient freudien. In Ecrits. Paris: Le Seuil. (Trabalho original publicado em 1960).

Mijolla-Mellor S. de. (2002). Le besoin de savoir. Paris: Dunod.

Mijolla-Mellor, S. de. (2005). Femmes, fauves et grands criminels. In S. de Mijolla-Mellor (dir.). La cruauté au féminin (pp. 23-54). Paris: PUF.

Platon (1992). Timée. In Timée, Critias. (trad. et présentation par Luc Brisson, pp. 95-310). Paris: GF Flammarion.

Rasmussen, K. (1974). Terre Wakan. Paris: Laffont.

Rouquet-Brutin, K. (2005). Archives de la cruauté chez Patricia Cornwell. In S. de Mijolla-Mellor (dir.), La cruauté au féminin (pp. 93-118). Paris: PUF.

Toro, G. C. (2001). Brennand: resonance and the univesality of the forms. Revista chilena de historia natural [online], 74(1), 183-194.

\section{Resumos}

(O sujeito entre sideração e gozo: a sublimação como solução. Sobre a obra de Francisco Brennand)

O presente artigo trata do universo inconsciente das esculturas do artista brasileiro Francisco Brennand (1927) e dos mecanismos arcaicos subjacentes à sua criação. Fundamentada sobre o tema da origem, esta pesquisa das obras de Brennand estuda as raízes do desejo sexual e sua relação aos objetos parciais, considerando a sideração do sujeito frente à crueldade de uma sexualidade arcaica e o gozo do artista como desafio à morte e ao enigma do nada. Ela se interessa então pelo processo da sublimação como transmutação do material traumático e pelo destino da pulsão escópica e de crueldade na criação artística.

Palavras-chave: Brennand, sublimação, origem, mito, gozo, pulsão escópica e de crueldade

(The subject between jouissance and total consternation: sublimation as a solution. Art of Francisco Brennand)

This article deals with the unconscious universe of the sculptures by the Brazilian sculptor Francisco Brennand (1927) and the archaic mechanisms that underlie their creation. Focused on the theme of origins this approach to Brennand's works studies the roots of sexual desire and its relation to partial objects, considering the subject's total consternation toward the cruelty of the artist's archaic sexuality and jouissance as a challenge to death and to the enigma of nothingness. The study then focusses on the sublimation process as transmutation of traumatic material and on the fate of the scopic drive and the drive toward cruelty in artistic creation.

Key words: Brennand, sublimation, origin, myth, jouissance, scopic and drive, cruelty drive 
(El sujeto entre sideración y gozo: la sublimación como solución. Sobre la obra de Francisco Brennand)

Este articulo trata del universo inconsciente de las esculturas del artista brasileño Francisco Brennand (1927) y de los mecanismos arcaicos subyacentes a su creación. Fundamentado en el tema del origen, este trabajo de investigación sobre las obras de Brennand estudia las raices del deseo sexual y su relación con los objetos parciales, teniendo en cuenta la sideración del sujeto frente a la crueldad de una sexualidad arcaica y el goce del artista como desafio a la muerte y al enigma de la nada. La investigación se orienta para el proceso de la sublimación como una transmutación del material traumático y por el destino de la pulsión escópica y de crueldad en la creación artística.

Palabras clave: Brennand, sublimación, origen, mito, gozo, pulsión escópica y de crueldad

(Das Subjekt zwischen Erstarrung und Genuss: die Sublimation als Lösung. Über das Werk von Francisco Brennand)

Dieser Beitrag behandelt das unbewusste Universum der Skulpturen des brasilianischen Künstlers Francisco Brennand (1927) und der archaischen Mechanismen, die seinen Werken zugrundeliegen. Diese Untersuchung von Brennands Werken gründet auf dem Thema des Ursprungs. Es werden die Wurzeln des sexuellen Begehrens und seine Beziehung zu den Teilobjekten untersucht. Dabei wird die Erstarrung des Subjektes gegenüber der Gewalt einer archaischen Sexualität und dem Genuss des Künstlers als Herausforderung des Todes und des Rätsels des Nichts beachtet. Die Untersuchung beleuchtet also den Sublimationsprozess als Transmutation des traumatischen Materials und das Schiksal des skopischen Triebes und der Grausamkeit im künstlerischen Schaffen.

Schlüsselwörter: Brennad, Sublimation, Ursprung, Mythos, Genuss, skopischer Trieb, Grausamkeit

Citação/Citation: Donard, V. (2014, setembro). Le sujet entre sidération et jouissance: À propos de l'œuvre de Francisco Brennand. Revista Latinoamericana de Psicopatologia Fundamental, 17(3-Suppl.), 638-651.

Editor do artigo/Editor: Prof. Dr. Manoel Tosta Berlinck 


\section{ARTIGOS}

Recebido/Received: 15.3.2014/ 3.15.2014 Aceito/Accepted: 15.4.2014 / 4.15.2014

Copyright: (C) 2009 Associação Universitária de Pesquisa em Psicopatologia Fundamental/ University Association for Research in Fundamental Psychopathology. Este é um artigo de livre acesso, que permite uso irrestrito, distribuição e reprodução em qualquer meio, desde que o autor e a fonte sejam citados / This is an open-access article, which permits unrestricted use, distribution, and reproduction in any medium, provided the original author and source are credited.

Financiamento/Funding: A autora declara não ter sido financiada ou apoiada / The author have no support or funding to report.

Conflito de interesses/Conflict of interest: A autora declara que não há conflito de interesses

/ The author has no conflict of interest to declare.

\section{VÉroniQue DoNARD}

Cátedra de pesquisas Francisco Brennand, Consulado francês no Recife, Universidade Católica de Pernambuco - Unicap (Recife, PE, Br); Pós-doutorado, Laboratório de Psicopatologia Fundamental na mesma Universidade; Doutora em psicopatologia, Universidade Paris-Diderot - Paris 7 (Paris, Fr); Graduação em psicologia clínica e psicopatologia, Universidade Paris-Diderot - Paris 7 (Paris, Fr).

Estrada do Encanamento, 702 - Casa Forte

52070-000 Recife, PE, Br

e-mail: veronique.donard@gmail.com 